[Vicino Oriente XXIV (2020), pp. 109-123]

\title{
FIGURE FEMMINILI E GESTIONE DEL POTERE DURANTE LA VI DINASTIA: LE DONNE DELLA CORTE DI PEPI I
}

\author{
Domizia D’Erasmo - Sapienza Università di Roma
}

\begin{abstract}
The kingdom of Pepi I (6 $6^{\text {th }}$ Dynasty) is characterized by a series of female characters who had important roles at the court of the king and, apparently, some of these seem to have played a part in the future of the Dynasty. In this paper, particular attention will be given to the cases of the anonymous bride of Pepi I, to Nebet vizier of Upper Egypt, to Ankhenespepi I, to Ankhenespepi II and to the role played by some princesses married to vizir or to high-ranking officers. The suggestionfounded on the analysis of epigraphic and archaeological evidence - is that these women were more than just vehicles for marriage alliances. In fact, what emerges is that several of these female characters, with the approval of the king, would exercise a power similar to that of their male counterparts.
\end{abstract}

Keywords: $6^{\text {th }}$ Dynasty; Pepi I; women study; royal women; administration

\section{INTRODUZIONE}

Al tramonto della V dinastia (2456-2297 a.C.), l'accentramento di potere nelle mani dei funzionari aveva cominciato a destare preoccupazione, poiché si venne probabilmente a creare un sistema di tipo feudale che minacciava il potere centrale. L'assenza di eredi maschi che potessero succedere a Unas rappresentò un punto cardine intorno al quale furono intessute le basi di cospirazioni che misero ulteriormente in crisi la casata regnante.

La VI dinastia (2297-2166 a.C.) fu per l'appunto caratterizzata da una serie di complotti orditi tra le mura del palazzo, e molto spesso da personalità vicine al sovrano, come ufficiali di alto rango o vizir. Ciò che emerge dalla documentazione archeologica ed epigrafica è un clima di forte instabilità, da imputare plausibilmente a macchinazioni volte a controllare la linea di successione al trono ${ }^{1}$. In quest'ottica, la componente femminile sembrò giocare un ruolo decisivo nell'ambito delle alleanze che legarono i sovrani ai loro ufficiali, o come nel caso di Pepi I, alle famiglie di governatori dei vari nomoi egiziani. Resta da chiedersi se il ruolo delle donne che gravitarono intorno alla corte di questi uomini fosse limitato a intessere delle relazioni di fiducia, che garantivano un controllo puntuale del Paese, oppure al tentativo di preservare la loro sicurezza.

All'inizio del suo regno, Pepi I, figlio di Teti - primo sovrano della VI dinastia, assassinato forse a seguito di una congiura portata a termine dai suoi vizir -, salì al trono con il nome di Nefersahor, ma successivamente lo modificò in Merira ${ }^{2}$. Egli regnò sull'Egitto per quarant'anni, dopo un breve periodo di regno da parte del fratello/fratellastro

1 Si ricorda in particolare l'assassinio di Teti documentato nelle epitomi degli Aegyptiaca di Manetone. Per una discussione sull'evento si rimanda a Kanawati 2002. Per un esame puntuale delle dinamiche genealogiche relative alla casata reale dell'Antico Egitto, con particolare riferimento all'Antico Regno, si rimanda a Baud 1999, 351-373 e passim.

2 Il coinvolgimento di alcuni vizir nell'omicidio di Teti sarebbe confermato dal fatto che i loro nomi furono erasi dalle pareti delle camere funerarie delle mastabe che essi avevano fatto costruire. Queste sepolture furono successivamente riassegnate, a conferma del fatto che questi personaggi avessero perso il loro potere e la loro posizione a corte. Per un approfondimento si rimanda a Kanawati 2002.

ISSN 0393-0300

e-ISSN 2532-5159

Rivista Open Access 
Userkara $^{3}$, considerato in alcuni casi come uno dei possibili partecipanti alla cospirazione contro il padre ${ }^{4}$. È noto che Pepi I sia salito al trono in giovane età e che per un certo lasso di tempo la regina Iput I, sua madre, sarebbe stata la reggente del trono d'Egitto facendone le veci.

$\mathrm{Si}$ attribuiscono con certezza sette spose al sovrano: sei furono seppellite nella sua necropoli a Saqqara, mentre l'esistenza della settima è documentata esclusivamente dall'Autobiografia di Uni ${ }^{5}$. Al di là delle unioni matrimoniali, è chiaro che la componente femminile ebbe una certa importanza durante il regno del sovrano. È noto che egli abbia protratto il sistema delle alleanze, sancite per mezzo di matrimoni che legavano ufficiali e vizir alla casata reale, già intrapreso dai suoi predecessori ${ }^{6}$. Tuttavia, con Pepi I, si assisterà al loro aumento esponenziale, probabilmente legato al momento di forte crisi in cui versava il potere centrale ${ }^{7}$.

In quest'ottica, il presente contributo mira ad affrontare un riesame delle principali figure femminili che ruotarono attorno alla corte di Pepi I. In particolare, si tenterà di offrire, attraverso un riesame della bibliografia pregressa e alla luce di nuovi studi, una panoramica sul ruolo rivestito dalle donne che di fatto contribuirono, insieme al sovrano, a plasmare il futuro della dinastia.

\section{DONNE ALLA CORTE DI PEPI I: UN APPROFONDIMENTO SU ALCUNE FIGURE FEMMINILI}

Che l'antica società egiziana riconoscesse alla sfera femminile uno status senza eguali, rispetto alla maggior parte delle culture antiche, è un fatto appurato ${ }^{8}$. Le donne avevano la possibilità di compiere atti legali, amministrare autonomamente la loro dote, ricevere delle eredità, rivestire importanti cariche amministrative e finanche governare il regno d'Egitto. Nonostante questa incoraggiante premessa, va sottolineato come molto spesso nell'ambito degli studi moderni che le riguardano, regine, principesse o importanti figure femminili di epoca faraonica, siano state quasi sempre considerate come un'appendice delle loro controparti maschili. Non è pertanto inopportuno citare le parole di Stefania Pignattari quando afferma che «tutte le regine caddero vittima di gender bias, pregiudizi, velati o

Il legame di parentela che intercorre tra Userkara e Teti non è del tutto chiaro (Kanawati 2002, 4; 157-158) Tuttavia, sembrerebbe che il passaggio tra il governo di Userkara e quello di Pepi I sia avvenuto in maniera pacifica (Grimal 1988, 102).

4 Grimal 1988, 102; Kanawati 2002,184-185.

5 L'Autobiografia di Uni (CGC 1435) è un prezioso documento epigrafico che racconta le vicende salienti della vita dell'ufficiale Uni al servizio di Teti, Pepi I e Nemtiemsaf I. Si rimanda per un approfondimento a Piacentini 1990. Si ricorda, inoltre, che il testo è attestato da una stele proveniente da Abido (CGC 1435) e da una sua 'copia' inscritta su due grandi blocchi in pietra rinvenuti dalla MAFS nella necropoli di Pepi I a Saqqara. Si rimanda per un approfondimento sul testo proveniente da Saqqara a Collombert 2015.

6 La più antica testimonianza di un matrimonio tra una delle figlie del sovrano e un personaggio di alto rango risale alla IV dinastia, e riguarda l'unione tra la principessa Khaamat, figlia di Shepseskaf, e il sommo sacerdote di Eliopoli, Ptahshepses. Si veda Callander 2006, 119-120.

7 Tyldesley 2006, 58-59.

8 Per quanto concerne lo status legale delle donne nell'Antico Egitto si rimanda a Johnson 1996, mentre per un approfondimento sulla sfera femminile si rimanda a Robins 1993. 
espliciti, che ancora sussistono e che distorcono la realtà dei fatti [...] con il risultato di relegare le protagoniste ai margini della Storia» ${ }^{9}$.

Il regno di Pepi I fu scandito da una particolare attenzione nei confronti della sfera femminile. È noto che il sovrano trasformò la mastaba destinata alla sepoltura della madre, la regina Iput $\mathrm{I}$, in una piramide, creando un santuario dalle fattezze di un tempio destinato al culto della donna, e includendo, per la prima volta, delle divinità nel programma iconografico del suo complesso funerario ${ }^{10}$. Come accennato nel paragrafo precedente, sono attribuite sette spose al sovrano, tuttavia è probabile che egli ne avesse in maggior numero, o come ipotizza Joyce Ann Tyldesley, una per ogni nomos d'Egitto ${ }^{11}$. Inoltre, come già era accaduto durante il regno di Teti, suo padre, le sorelle di Pepi I si unirono principalmente a ufficiali di alto rango e vizir, mentre per almeno due delle sue figlie, Neith e Iput II, è certo il matrimonio con Pepi II, anch'esso figlio di Pepi I e perciò fratello delle due giovani.

I matrimoni contratti da Pepi I furono celebrati all'insegna di nuove alleanze con l'Alto e il Basso Egitto. Si è già accennato alla situazione di instabilità in cui versava la casata reale quando il giovane sovrano salì al trono. A tal proposito, il primo periodo in cui egli governò dovette risentire ampiamente degli avvenimenti che precedettero la sua ascesa al trono: la lotta per la successione in seguito alla morte di Unas e l'assassinio di Teti. È in questo periodo che viene generalmente collocata la vicenda del "complotto reale" ordito da una delle spose di Pepi I, documentato esclusivamente dall'Autobiografia di Uni. Infatti, è solo grazie a questa testimonianza che può essere compiuto un viaggio a ritroso che conduce alle prime fasi del regno del sovrano, volto a individuare quello che generalmente è considerato l'evento chiave che diede vita alle future alleanze matrimoniali di Pepi I, che di fatto furono la fortuna della dinastia: i matrimoni abideni.

Ciò che va sottolineato, è che le figure femminili che si susseguirono nella VI dinastia, e in particolare durante il regno di Pepi I, non solo furono indispensabili per gestire la situazione, ormai fuori controllo, legata all'accentramento di potere nelle mani dei nomarchi, ma contribuirono, inoltre, a creare delle condizioni favorevoli alla continuità dinastica, svolgendo quasi la funzione di garanti delle sorti del regno.

\subsection{La questione della sposa anonima}

Molteplici studi furono indirizzati a comprendere le dinamiche che si celarono dietro al complotto della controversa figura della sposa anonima di Pepi $\mathrm{I}^{12}$. Non è nell'interesse di questo contributo arrivare a delineare le motivazioni del suo tradimento, tuttavia sembra opportuno per l'argomento trattato in questa sede affrontare un breve riesame delle notizie riguardanti la donna.

Pignattari 2008, 11; Callander 1998, 45.

Callander 2015, 40-41.

Tyldesley 2006, 59

2 Per quanto concerne le questioni del complotto dell'harem e del successivo processo alla sposa anonima di Pepi I, citato nell'Autobiografia di Uni, si rimanda a Goedicke 1954, 181-183; Goedicke 1955; Roccati 1982, 190 e cfr. con Baud 1999, 626:280. 
Innanzitutto, va chiarita la questione relativa al nome della regina, poiché questa viene identificata con appellativi diversi nell'ambito di più pubblicazioni scientifiche. Nell'Autobiografia di Uni (CGC 1435) si fa riferimento alla donna come "la moglie del re,

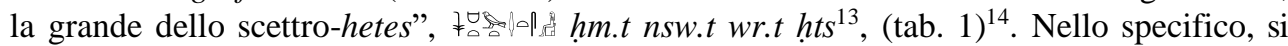
riscontra un fraintendimento generale del titolo caratteristico delle regine dell'Antico Regno, wr.t-hts ${ }^{15}$, interpretato come nome proprio della donna, che quindi viene spesso chiamata erroneamente Weret-Iamtes, Iamtes oppure Amtes ${ }^{16}$. Inoltre, il titolo onorifico "la grande dello scettro-hetes", è attestato per altre spose di Pepi I (tab. 3, tab. 4), e per tanto non deve essere considerato come peculiare della congiurante ${ }^{17}$.

Il fatto che nell'Autobiografia di Uni la donna fosse identificata con un titolo che la contraddistingueva ancora come regina, fa sicuramente riflettere su ciò che si voleva far trasparire dell'evento. È chiaro, in assenza di ulteriori testimonianze, che la donna subì una damnatio memoriae e lo stesso può essere supposto per i suoi discendenti.

\begin{tabular}{|l|l|l|l|}
\hline \multicolumn{4}{|c|}{ Titolatura sposa anonima } \\
\hline Provenienza & Titolo & Traslitterazione & Traduzione \\
\hline CGC 1435 & $\ddagger 0$ & hm.t nsw.t & Moglie del Re \\
\hline CGC 1435 & wr.t hts & Grande dello scettro-hetes \\
\hline
\end{tabular}

Tab. 1 - Titolatura della sposa anonima ricavata dall'Autobiografia di Uni (CGC 1435). La traduzione dei titoli onorifici si basa su quella riportata dal Wörterbuch der ägyptische Sprache, ed è stata confrontata con quella presente in Jones 2000.

\subsection{Nebet, vizir dell'Alto Egitto}

Moglie di $h w i$, Khui, nobile residente ad Abido ${ }^{18}$, $\sigma \downarrow \bullet b . t^{19}$, Nebet, vizir dell'Alto Egitto (tab. 2), amministrò il Paese durante il regno di Pepi I assieme al marito. Ella fece

13 Le traslitterazioni e traduzioni fornite qui e altrove nel presente articolo sono quelle riportate nel Wörterbuch der ägyptischen Sprache $(W b)$, ulteriormente confrontate con quelle riportate in Jones 2000 e nel Lexikon der $\ddot{A}$ gyptologie $(L \ddot{A})$. In caso contrario si esplicitano i riferimenti in nota.

14 Baud 1999, 626:280. Il titolo onorifico della donna non è attestato nel testimone dell'Autobiografia di Uni proveniente dalla necropoli di Saqqara, che tuttavia menziona l'evento della cospirazione dell'harem. Si presuppone che il titolo fosse riportato nella riga superiore del testo che presenta grandi lacune. Per un approfondimento si rimanda a Collombert 2015, 148

15 Sebbene alcuni studiosi preferiscano tradurre il titolo wr.t hts con 'Grande favorita', in questa sede si è deciso di seguire la traduzione riportata nel Wörterbuch der ägyptischen Sprache: la "grande dello scettro-hetes". Per un approfondimento si rimanda a $W b$ 1,80.6; 3, 202.9-12; L̈̈ VII, 435 e Goedicke 1954, 88:2. Si segnala la bibliografia sulle attestazioni del titolo onorifico in Jones 2000, 403:1481.

16 Bresciani 1969, 19; Lichtheim 1975, 18-23; Tyldesley 2006, 57; Tresson 1919, 10.

17 Tyldesley 2006, 57.

18 La sepoltura di Khui è generalmente individuata nella necropoli di Teti a Saqqara, tuttavia alcuni studiosi tra cui Naguib Kanawati sembrano incerti sul fatto che questa sia stata realmente utilizzata per ospitare il corpo del vizir dell'Alto Egitto. Il dubbio nasce dal fatto che la tomba di Saqqara apparirebbe di fattezze eccessivamente umili per un uomo di tale statura sociale, cosa che ha portato a presuppore che non fu mai utilizzata per Khui. Un'ipotesi è che l'uomo fosse stato in realtà seppellito ad Abido, sua città natale, e dalla quale esercitava il ruolo di vizir. Per ulteriori informazioni si consulti Kanawati 2002, 93. 
ingresso nella famiglia reale all'indomani del matrimonio delle figlie con il sovrano ${ }^{20}$. Lo stesso avvenimento segnò inoltre l'ascesa politica dell'aristocrazia di Abido, poiché dall'unione tra Pepi I e le due giovani di cui sopra, nacquero Nemtiemsaf I e Pepi $\mathrm{II}^{21}$, successori al trono del sovrano.

Sebbene la donna rivestisse il ruolo di vizir assieme al compagno, ella fu certamente investita di onori maggiori rispetto all'uomo, come è deducibile dalle testimonianze epigrafiche che la riguardano (CGC 1431; CGC 1575; CGC 157822). È probabile infatti, che le titolature con le quali venne designata, furono "costruite" appositamente per elevare il suo status non di sangue regale dopo che ella divenne a tutti gli effetti suocera del sovrano e membro della famiglia reale (tab. 2$)^{23}$.

Il motivo della disparità tra i titoli attribuiti alla donna e quelli riservati al marito è individuabile secondo Henry George Fisher nelle dinamiche del rapporto tra Nebet e Pepi $\mathrm{I}^{24}$. Secondo lo studioso, è probabile che la donna abbia con il tempo stretto un legame particolare con il sovrano, abbastanza rilevante da valerle l'attribuzione di onorificenze e privilegi maggiori rispetto a Khui ${ }^{25}$. Questa interpretazione offre senza dubbio una spiegazione esaustiva all'influenza che Nebet ebbe alla corte di Pepi I, tuttavia, alla luce delle nuove scoperte effettuate nel complesso funerario della di lei figlia, Ankhenespepi II (§4.), potrebbe essere considerata altrettanto valida l'ipotesi per cui gli onori attribuitegli furono in realtà una conseguenza del legame che essa aveva con la regina, e non con il sovrano (§§ 2.3., 3.).

19 Mariette 1880, 86-87. Per un ulteriore approfondimento su Khui, Nebet, e sui membri della loro famiglia, si rimanda a Baud 1999, 629-631.

20 Goedicke 1955, 180-183. È possibile che Nebet fosse la madre della sola Ankhenespepi II e che Ankhenespepi I fosse figlia di una prima moglie di Khui. Si rimanda per un approfondimento a Fischer 1976, 75:40.

21 Si è ancora incerti sulla durata del regno di Nemtiemsaf I. L'attestazione più tarda proviene da un'iscrizione lacunosa della cava di Hatnub, e testimonia il raggiungimento del quinto anno di censimento da parte del sovrano. Al contrario, il regno di Pepi II è bene attestato nella maggior parte delle liste dei sovrani, e si aggira più o meno intorno ai 30 anni di governo.

22 La stele CGC 1578 fu probabilmente realizzata durante i primi anni di regno di Pepi II, e quindi durante il periodo di co-reggenza di Ankhenespepi II (Fisher 2000, 37; n. 237).

23 Per un approfondimento sulla titolatura di $n b . t$ in CGC 1578: Fischer 1976, 74-75. Inoltre, per il titolo $\underline{h} k r . t$ $n s w . t$ si rimanda a $W b 3,401.6-11$; Jones 2000, 794:2899; per il titolo šps.t nsw.t si rimanda a $W b 4$, 450.1; Jones 2000, 990:3664; per il titolo iry.t-p'.t si rimanda a Jones 2000, 338:1247; Wb 2, 415.15-416.6; per il titolo h3.tt-' si rimanda a $W b 3,26.3-5$; Jones 2000, 496:1858; per il titolo s3.t $m r h w$ si rimanda a $W b 2,112.6$;

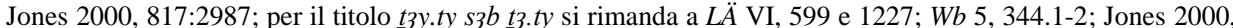
1000:3706; per il titolo s3.t $\underline{d}$ hwty si romanda a Jones 2000, 824:3010; per il titolo smr.t bi.t si rimanda a Wb 4, 138.5-139.5; Jones 2000, 897:3292; per il titolo s3.t hr si rimanda a Jones 2000, 824:3007.

24 Per quanto concerne l'iconografia, continuano ad essere applicati i consueti canoni stilistici tipici dell'Antico Egitto, per tanto Khui è sempre rappresentato di dimensioni maggiori rispetto a Nebet sebbene questa sia accompagnata da un maggior numero di titoli onorifici.

25 Fischer 2000, 37 


\begin{tabular}{|c|c|c|c|}
\hline \multicolumn{4}{|c|}{$\begin{array}{c}-3 \sqrt{ } \\
\text { Titolatura di Nebet }\end{array}$} \\
\hline Provenienza & Titolo & Traslitterazione & Traduzione \\
\hline CGC 1431 & $\neq \hat{\imath}$ & $\underline{h} k r . t n s w . t$ & L'ornamento del Re \\
\hline CGC 1575 & $\neq 0$ & šps.t nsw.t & La venerabile del $\mathrm{Re}$ \\
\hline CGC 1578 & D० & iry.t-p $\cdot t$ & Principessa ereditaria \\
\hline CGC 1578 & 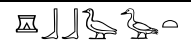 & s3.t $g b b$ & Figlia di Geb \\
\hline CGC 1578 & 90 & ha.tt-' & Contessa \\
\hline CGC 1578 & 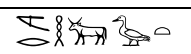 & s3.tmrhww & Figlia di Mnevis \\
\hline CGC 1578 & 政的为 & t3y.ty s3b tz.ty & Vizir \\
\hline CGC 1578 & 乐 30 & s3.t d dhwty & Figlia di Thot \\
\hline CGC 1578 & 结防品 & smr.t bi.t & $\begin{array}{l}\text { Amica unica del Re del Basso } \\
\text { Egitto }\end{array}$ \\
\hline CGC 1578 & 是是。 & s3.t $h r$ & Figlia di Horus \\
\hline
\end{tabular}

Tab. 2 - La titolatura di Nebet è stata ricavata dalle informazioni riportate in Fischer 1976, 74-75; 1977, 141; Mariette1880, 86-87; 1869, pl. II. La traduzione dei titoli onorifici si basa su quella riportata dal Wörterbuch der ägyptische Sprache, ed è stata confrontata con quelle presenti in Jones 2000 e nel Lexikon der Ägyptologie.

\subsection{Ankhenespepi I e Ankhenespepi II}

Parte delle informazioni relative ad Ankhenespepi I e Ankhenespepi II possono essere estrapolate da un'iscrizione proveniente dalla mastaba di loro fratello (o fratellastro), nonché vizir dell'Alto Egitto, $2 \$ \underline{d} \underline{d}^{\prime} w$, Giau $\left(\mathrm{CGC} 1431^{26}\right)$. Questa è una delle principali testimonianze attraverso cui si è a conoscenza del fatto che le due donne diedero alla luce i successori al trono di Pepi I: Nemtiemsaf I e Pepi II. Tuttavia, dall'iscrizione non pervengono ulteriori informazioni circa il periodo in cui il matrimonio delle sorelle fu celebrato, né a proposito della loro età ${ }^{27}$. Certo è che generalmente $\mathrm{i}$ così detti matrimoni abideni vengono imputati al bisogno che Pepi I ebbe di rafforzare i rapporti con il Medio e l'Alto Egitto, e quindi di riacquisire il pieno controllo dei traffici commerciali in tutto il Paese $^{28}$.

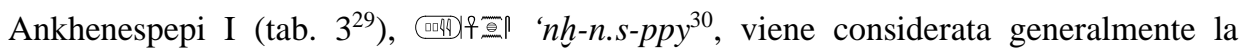
madre di Nemtiemsaf I, tuttavia l'opinione di Hans Goedicke è che quest'ultimo sovrano e

26 Una bibliografia completa sulle pubblicazioni relative alla stele CGC 1431 è disponibile in Fischer 1977, 141143.

27 Goedicke 1955, 181.

28 Grimal 1988, 104-105.

29 Per un approfondimento sul titolo onorifico $h m . t$ nsw.t si rimanda a $W b$ 3, 77-78.13; per il titolo wr.t hts Cfr. n. 15; per il titolo wr.t hsw.t si rimanda a Wb 3, 158.8-11; LÄ III, 473; Jones 2000, 401:1478; per il titolo ht wr si rimanda a Jones 2000, 755:2752; Wb 3, 344.7; per il titolo smr.t hr si rimanda a $W b 4$, 139.6; Jones 2000, 897:3293; per il titolo tis.t $h r$ si rimanda a Wb 5, 242.11; Jones 2000, 1001:3711; per il titolo $m$ w.t nsw.t-bi.t si rimanda a $W b$ 2, 331.4; Jones 2000, 426:1576. Si rimanda a Baud 1999, 426-428:37 per un ulteriore approfondimento su Ankhesenpepi I.

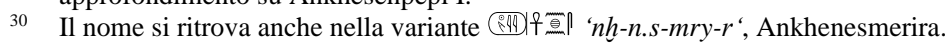


Pepi II non fossero affatto fratelli, bensì fratellastri. Secondo lo studioso infatti, Nemtiemsaf I sarebbe stato figlio della regina anonima di Pepi I, e Ankhenespepi I avrebbe rivestito il solo ruolo di madre dinastica del sovrano ${ }^{31}$. Considerando quest'ipotesi, Nemtiemsaf I sarebbe stato un uomo adulto alla morte del padre, e non un adolescente come spesso viene considerato.

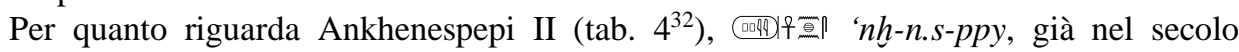
scorso una statuetta in alabastro - forse proveniente da Elefantina - che la raffigurava in trono nell'atto di sostenere sulle ginocchia il giovane Pepi II (fig. 1.), aveva fatto supporre che la donna vantasse un legame particolarmente stretto con il figlio, e soprattutto che si guardasse a lei con estremo rispetto ${ }^{33}$.

\begin{tabular}{|c|c|c|c|}
\hline \multicolumn{4}{|c|}{ 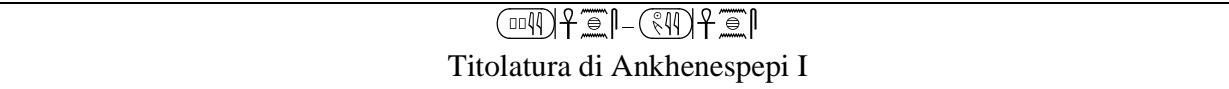 } \\
\hline Provenienza & Titolo & Traslitterazione & Traduzione \\
\hline CGC 1431 & $\neq 0$ & ḩm.t nsw.t & Moglie del Re \\
\hline CGC 1431 & $\int_{\frac{1}{a}}$ & wr.t ḥts & Grande dello scettro-hetes \\
\hline CGC 1431 & $\sqrt{1}$ & wr.t hasw.t & Grande di lode \\
\hline CGC 1431 & 为 & $h t w r$ & Accolita del Grande \\
\hline CGC 1431 & Anto & smr.t hrr & Amica di Horus \\
\hline CGC 1431 & ADo & tis.t ḥr & Compagna di Horus \\
\hline CGC 1431 & 誌监 & mw.t nsw.t-bi.t & Regina Madre \\
\hline
\end{tabular}

Tab. 3 - La titolatura di Ankhenespepi I è stata ricavata dalle informazioni riportate in Mariette 1869(tav. II). La traduzione dei titoli onorifici si basa su quella riportata dal Wörterbuch der ägyptische Sprache ed è stata confrontata con quella presente in Jones 2000.

Dal complesso funerario di Ankhenespepi II proviene una seconda iscrizione, lacunosa, incisa su di un blocco di pietra calcarea, forse pertinente a uno stipite, che sembra confermare la possibilità di una co-reggenza da parte della donna: "Sua Maestà l'ha fatto (quello) [per] lei mentre era nella Residenza"34. Considerando che il complesso funerario della regina fu costruito e decorato durante il regno di Pepi II, è proprio a questo sovrano che il testo fa riferimento. Secondo Philippe Collombert, direttore della Mission archéologique française de Saqqâra (MAFS) ${ }^{35}$, il sottolineare che la donna si trovasse nella Residenza corrisponderebbe a un'affermazione implicita del fatto che Ankhenespepi II

31 Goedicke 1955, 183.

32 Per un approfondimento sulla titolatura di Ankhenespepi II Cfr. n. 29. Inoltre, per il titolo onorifico sz.t ntr $r$ si rimanda a Jones 2000, 822:3004. Si rimanda a Baud 1999, 428-429:38 per un ulteriore approfondimento su Ankhesenpepi II.

33 Tyldesley 2006, 61. La statuetta di Ankhenespepi II è datata all'incirca al 2288-2224/2194 a.C. (Brooklyn Museum, Charles Edwin Wilbour Fund, 39.119).

34 Collombert 2018, 74.

35 Per ulteriori informazioni sulla Mission archéologique française de Saqqâra (MAFS) si rimanda al sito web della missione: http://mafssaqqara.wixsite.com/mafs. 
governasse il Paese per conto del giovane Pepi II. A tal proposito, si ricorda che non è di certo inusuale per gli antichi egizi ricorrere a parafrasi per esprimere dei concetti per cui non esistevano termini specifici, ed è noto che il reale esercizio del potere da parte di una regina-madre non si formalizzò mai come schema ideologico durante l'epoca faraonica ${ }^{36}$.

Un'ulteriore conferma del potere che esercitò la donna è data dalla monumentalità del suo complesso funerario, più grande rispetto a quello delle altre regine e principesse, ospitava obelischi di circa 5 metri di altezza, quando consuetamente, quelli situati dinanzi alle piramidi delle altre donne della famiglia reale non raggiungevano i 2 metri ${ }^{37}$. Le pareti della camera funeraria della donna erano decorate con i Testi delle Piramidi, e il sarcofago che la ospitava, trafugato ab antiquo, aveva le stesse caratteristiche di quello riservato ai sovrani ${ }^{38}$.

Ella avrebbe pertanto tenuto le redini del regno in seguito alla morte di Pepi I, probabilmente deceduto pochi anni dopo la loro unione, ed è ipotizzabile che, amministrando il regno per conto del giovane figlio, riuscì a infondere maggiore potere anche alla casata di Abido, suo luogo natìo, e per estensione alla sua famiglia.

\begin{tabular}{|c|c|c|c|}
\hline \multicolumn{4}{|c|}{ 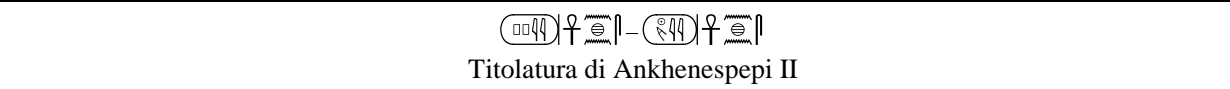 } \\
\hline Provenienza & Titolo & Traslitterazione & Traduzione \\
\hline CGC 1431 & $\neq 0$ & hm.tnsw.t & Moglie del Re \\
\hline CGC 1431 & $\lim _{0}$ & wr.t hts & Grande dello scettro-hetes \\
\hline CGC 1431 & $\sqrt{\frac{1}{0}}$ & wr.t hsw.t & Grande di lode \\
\hline CGC 1431 & 930 & s3.tntr & Figlia del Dio \\
\hline CGC 1431 & 有早 & $h t w r$ & Accolita del Grande \\
\hline CGC 1431 & Nito & smr.t hr & Amica di Horus \\
\hline CGC 1431 & MDO & tis.t hr & Compagna di Horus \\
\hline CGC 1431 & 箇 0 & mw.t nsw.t-bi.t & Regina Madre \\
\hline
\end{tabular}

Tab. 4 - La titolatura di Ankhenespepi II è stata ricavata dalle informazioni riportate in Mariette 1869, pl. II. La traduzione dei titoli onorifici si basa su quella riportata dal Wörterbuch der ägyptische Sprache ed è stata confrontata con quella presente in Jones 2000.

\subsection{Il ruolo delle principesse}

Analizzando le iscrizioni provenienti dalle necropoli dei sovrani della VI dinastia, e in particolare delle sepolture riservate a funzionari di alto rango e vizir, si può riscontrare che in molti casi si ricorse alla pratica di contrarre matrimoni con donne della famiglia reale. È già stata descritta nei paragrafi precedenti la politica di alleanze matrimoniali che caratterizzò questo periodo storico, tuttavia ancora una volta appare molto più marcato

6 Collombert 2018, 75.

37 Collombert 2018, 74.

38 Dobrev et al. 2010, 279. 
l'interesse nei confronti dei compagni di queste donne, piuttosto che a riguardo del ruolo che esse detennero.

Naguib Kanawati ha messo in evidenza il coinvolgimento di alcuni vizir nel complotto ordito contro Teti, ipotizzando una loro implicazione sulla base dell'analisi dei danneggiamenti volontari all'interno delle loro sepolture. Furono almeno cinque i nomi di ufficiali erasi dalle iscrizioni nelle mastabe della necropoli di Teti ed è possibile riscontrare su altre sepolture l'accanimento volontario contro le rappresentazioni di vizir o ufficiali ${ }^{39}$.

Dalle iscrizioni pertinenti a queste mastabe traspare, inoltre, che tra gli uomini sopracitati molti sposarono una figlia del re (fig. 2$)^{40}$, e per alcuni si presuppone l'esistenza di matrimoni precedenti sebbene il nome delle prime mogli, laddove siano esistite, non è riportato. È interessante sottolineare come in questi contesti le figure delle principesse non furono apparentemente scalfite, né i loro nomi erasi, a differenza di quanto accadde per i loro mariti, e talvolta figli ${ }^{41}$. Risulta difficile immaginare, alla luce di quanto detto nei paragrafi precedenti, che queste donne fossero totalmente all'oscuro delle macchinazioni che avvenivano in seno alle loro famiglie. D'altra parte, sono noti fenomeni di accanimento anche nei confronti di figure femminili al tempo della VI dinastia: ne è un esempio il danneggiamento del volto della regina Ankhenespepi I in un frammento (E-0192) dal tempio mortuario di Pepi I, e il danneggiamento del volto della regina Mehaa, il cui trattamento può essere messo in correlazione con quello riservato a suo figlio, il principe ereditario Hornetierkhet ${ }^{42}$. Secondo Vivienne Gae Callander, queste azioni volte a mutilare le figure delle regine, combinate alle probabili discordie per la successione al trono, potrebbero essere un sintomo del perpetuarsi di contese all'interno della famiglia reale ${ }^{43}$.

Sebbene la paternità delle figlie dei sovrani sia generalmente più che certa - salvo alcuni casi particolari _ ${ }^{44}$, la loro maternità risulta più difficile da attribuire. Pertanto, ipotizzando che parte di queste donne dovette discendere da una delle così dette "regine secondarie", è possibile immaginare che, qualora si fossero create le circostanze per la messa in atto di complotti volti a spodestare i legittimi eredi al trono d'Egitto, esse avrebbero potuto approfittare dell'occasione. Tuttavia, in assenza di prove concrete che dimostrino una partecipazione attiva delle principesse alle contese delle casate reali, non

39 Kanawati 2002, 166-167.

40 I titoli onorifici più diffusi per le principesse, indicativi del loro lignaggio, erano s3.t nsw.t (Wb $3,412.7 ; L \ddot{A}$ III, 659; Jones 2000, 817:2988) e s3.t nsw.t n.t ht.f (Wb 3, 412.5.7; Jones 2000, 819:2993). Per un approfondimento sui titoli s3.t nsw.t e s3.t nsw.t n.t ht.f si rimanda a Baud 1999, 162-189 e Callander 2006, 124-126.

41 È opportuno tenere presente che non sono attestate, fino ad ora, sepolture individuali di donne all'interno della necropoli di Teti a Saqqara. L'unica eccezione è quella di Negietempet, madre del vizir Mereruka, marito di una delle figlie di Teti (fig. 2.). Sono ancora da chiarire le motivazioni che valsero il privilegio di avere una sepoltura personale alla donna. Si rimanda per un approfondimento sulla sepoltura di Negietempet a Kanawati 2002, 107.

42 Callander 2015, 48

43 Callander 2015, 48

44 Ad esempio, per quanto riguarda le figlie di Teti, vi è in alcuni casi l'incertezza sull'attribuirne la paternità a questo sovrano piuttosto che a Unas. Per ulteriori informazioni si rimanda a Kanawati 2002. Si ricorda inoltre, per il Medio Regno, il caso emblematico della principessa Neferuptah, una delle figure femminili più interessanti della XII Dinastia, la cui discendenza da Amenemhat III è ancora fonte di dibattito nel mondo accademico. Si rimanda a Pignattari 2008, 53-68 per una sintesi esaustiva e la bibliografia sull'argomento. 
resta che chiedersi se queste fossero totalmente estranee a ogni tipo di macchinazione perpetuata dai componenti delle proprie famiglie.

\section{CONCLUSIONE}

Se si guarda alle celebri liste di sovrani dell'Antico Egitto, è chiaro, in virtù di quanto detto nei paragrafi precedenti, che queste, seppur menzionino delle regine che governarono il Paese, sono del tutto insufficienti a rivelare la pluralità di eventi che ebbero vita all'interno delle residenze regali. Che le notizie relative alle vite di queste donne giungano a noi sotto forma di debole eco non è certo fonte di svalutazione delle loro figure. In una società in cui le donne non erano costrette a vivere necessariamente all'ombra degli uomini, sembra più che lecito attribuire a queste personalità le stesse ambizioni delle loro controparti, seppur nell'accezione negativa che le connota come cospiratrici. Pertanto, più che enfatizzare il ruolo di Nebet ( $\$ 2.2$.), di Ankhenespepi I e II (§ 2.3.), della sposa anonima ( $\$ 2.1$.), o delle molteplici principesse ( $\$ 2.4$.), questo dovrebbe essere uniformato a quello delle loro controparti, applicando una chiave di lettura che non le releghi ai margini degli eventi dinastici e che non le connoti come 'eccezioni'.

E stato messo in evidenza come le dinamiche di gestione del potere alla corte di Pepi I possano essere riconsiderate alla luce delle nuove scoperte archeologiche condotte nella necropoli del sovrano a Saqqara (§ 2.3.). In particolare, il riesame di alcune delle principali figure femminili che ruotarono intorno alla sua corte, ha messo in evidenza il fatto che queste donne avessero un'importanza più che secondaria nel corso della VI dinastia, a dispetto di ciò che può trasparire dalla tradizionale documentazione.

Compiendo un percorso diacronico attraverso il regno di Pepi I si può notare che fin dall'inizio del suo governo ci fu da parte del sovrano un'attenzione particolare nei confronti della figura della madre, la regina Iput I, manifestata attraverso la costruzione del suo magnifico complesso funerario. In questo stesso periodo, dovettero continuare a partecipare alla vita di corte le sorelle di Pepi I, che avevano contratto matrimonio con membri di spicco della società durante il regno di Teti. Questi uomini, secondo l'opinione, supportata dalle testimonianze archeologiche, di Naguib Kanawati, sarebbero stati puniti a seguito di crimini volti a capovolgere le sorti del regno ${ }^{45}$. Sembra poco probabile, in virtù di quanto riportato sopra, non presupporre nessun coinvolgimento delle loro spose nell'ambito delle congiure che essi ordirono.

L'Autobiografia di Uni riporta l'implicazione di una donna nell'ambito della cospirazione dell'harem reale di Pepi I. In questo testo è riportato che il processo alla sposa anonima avvenne in segreto, e che Uni fu l'unico testimone e giudice, assistito da uno scriba di Hierakompolis. Il fatto che l'ufficiale sottolinei la segretezza dell'evento è in linea con quanto tramandato dalla documentazione testuale pertinente all'Antico Regno. Per questo periodo infatti, gran parte delle informazioni relative a complotti/tradimenti proviene da documenti più tardi, come è il caso delle epitomi degli Aegyptiaca di Manetone, mentre per i periodi successivi sono attestate delle testimonianze più eloquenti

45 Kanawati 2002, 157-176. 
coeve al periodo degli avvenimenti ${ }^{46}$. In relazione al regno di Pepi I, è da considerare che la sposa anonima fu condannata, e che subì una damnatio memoriae, tuttavia l'avvenimento del processo, stando alle fonti, fu in qualche modo occultato ai membri della corte reale, sebbene non possa sicuramente essere passato inosservato all'interno dello stesso harem, e a coloro che vi risiedevano o lavoravano.

Per quanto concerne i tentativi di sovvertire l'ordine nell'arco della VI dinastia, questi non dovettero essere unicamente indirizzati all'accentramento di potere nelle mani dei soli ufficiali e vizir di sesso maschile. Una conferma di ciò che poteva implicare nella vita di queste donne il raggiungimento del potere da parte del figlio di una regina è testimoniato dalla figura di Ankhenespepi II. È ipotizzabile che la donna, divenuta regina giovanissima in seguito al matrimonio con Pepi I, ormai giunto alla fine del suo regno, abbia potuto esercitare, in quanto madre del futuro sovrano d'Egitto, Pepi II, e in quanto moglie del suo predecessore, Nemtiemsaf I, un potere superiore a quello delle altre spose, rendendo possibile la gestione del regno da parte di figure femminili che non siano a tutti gli effetti sovrani. A tal proposito, non è un caso che la madre di Ankhenespepi II, Nebet, sia stata investita di onori considerevoli che l'hanno caratterizzata come una delle donne più rilevanti dell'Egitto faraonico, o che Giau, suo fratello, abbia ricoperto il ruolo di vizir come i suoi genitori. È pertanto ipotizzabile che, per estensione, anche le principesse giocassero il loro ruolo per il raggiungimento di maggior potere, allo stesso modo della sposa anonima, tuttavia, in assenza di ulteriori testimonianze, questa resta un'ipotesi che meriterebbe un approfondimento.

In conclusione, si sottolinea come sia piuttosto riduttivo pensare a regine, principesse o donne di alto rango come meri veicoli di alleanze atte a consolidare la politica del regno d'Egitto. Ma, ancora una volta, citando le parole di Stefania Pignattari «il tipo di pregiudizio più ricorrente e insidioso è quello che associa l'ascesa al trono di una donna a un momento di crisi dello Stato o della dinastia, come se solo condizioni estreme potessero consentire un avvenimento altrettanto estremo ${ }^{47} \gg$. In particolare, alla luce degli avvenimenti che caratterizzarono il regno di Pepi I, e dei suoi predecessori e successori, appare evidente che, anche laddove le testimonianze relative alle donne si fanno più rade rispetto a quelle degli uomini, queste contribuiscono a ricostruire una partecipazione più che attiva della componente femminile alla gestione del regno e al futuro della dinastia (fig. 2.), la cui comprensione è ancora del tutto in fieri nell'ambito degli studi egittologici.

\footnotetext{
46 Per il Nuovo Regno si ricorda il caso simile a quello avvenuto alla corte di Pepi I del complotto avvenuto nell'harem reale contro Ramesse III, in cui il processo fu portato avanti da dodici giudici, e le punizioni che furono inflitte alla congiurante - una sposa secondaria del sovrano - e agli altri partecipanti, furono trascritte con accuratezza su un documento papiraceo, il cosiddetto Papiro Giudiziario di Torino. Per ulteriori informazioni si rimanda a Müller-Wollermann 2015.

47 Pignattari 2008, 11-12.
} 


\section{ELENCO DELLE ABBREVIAZIONI}

CGC L. Borchardt (ed.), Catalogue général des antiquités égyptiennes du musée du Caire. Denkmäler des alten reiches (ausser den statuen), vols. II, Berlin 1937-1964.

LÄ W. HELCK - E. OTTO - W. WESTENDORF (eds.), Lexikon der Ägyptologie, vols. VII, Wiesbaden 1975-1992.

MAFS Mission archéologique française de Saqqâra, http://mafsaqqara.wixsite.com/mas, ultima consultazione effettuata il 29/5/2020.

Wb A. ERMAN - W. GRAPOW (eds.), Wörterbuch der ägyptische Sprache, vols. VII, Leipzig 1926-1931.

\section{BIBLIOGRAFIA}

BAUD, M.

$1999 \quad$ Famille royale et pouvoir sous l'Ancien Empire égyptien, vols. II, Le Caire 1999.

BRESCIANI, E.

1969 Letteratura e poesia dell'Antico Egitto, Torino 1969.

CALLANDER, V.G.

1998 What Sex was King Sobek-Neferw?: A Modern Journal of Ancient Egypt 9/1 (1998), pp. 45-56.

2006 The Iconography of the Princess in the Old Kingdom: M. BÁRTA (ed.), The Old Kingdom Art and Archaeology. Proceedings of the conference held in Prague, may 31 - June 4, 2004, Prague 2006, pp. 119-126.

2015 Some Sixth Dynasty Queens: an historical perspective: M. BARTA - F. COPPENS - J. KREJCI (eds.), Abusir and Saqqara in the year 2015, Prague 2015, pp. 39-50.

COLLOMBert, $\mathrm{P}$.

2015 Une nouvelle version de l'autobiographie d'Ouni: R. LEGROS (ed.), Cinquante ans d'éternité. Jubilé de la Mission archéologique française de Saqqâra (1963-2013), Le Caire 2015, pp. 145-157.

2018 Recent discoveries of the Mission archélogique franco-suisse de Saqqâra in the funerary complex of Queen Ankhnespepy II: Saqqara Newsletter 16 (2018), pp. 66-75.

Dobrev, V. - Labrousse, A. - Mathieu, B. - Miniault-Gout, A. - Janot, F.

2010 Le dixième pyramide à textes de Saqqâra: Ânkhesenpéppy II. Rapport préliminaire de la campagne de fouilles 2000: Bulletin de l'Institut Français d'Archéologie Orientale 100 (2010), pp. 275-296

Dodson, A. - HiLTON, D.

2010 The Complete Royal Families of Ancient Egypt, London 2010.

FISCHER, G.

1976 Varia, New York 1976.

1977 Orientation of hieroglyphs II, part. I: reversals, New York 1977.

2000 Egyptian women of the Old Kingdom and of the Heracleopolitan Period, New York 2000. GOEDICKE, $\mathrm{H}$.

1954 An approximate date for the harem investigation under Pepy I: Journal of the American Oriental Society 74 (1954), pp. 88-89.

1955 The Abydene Marriage of Pepy I: Journal of the American Oriental Society 75 (1955), pp. 180-183. 


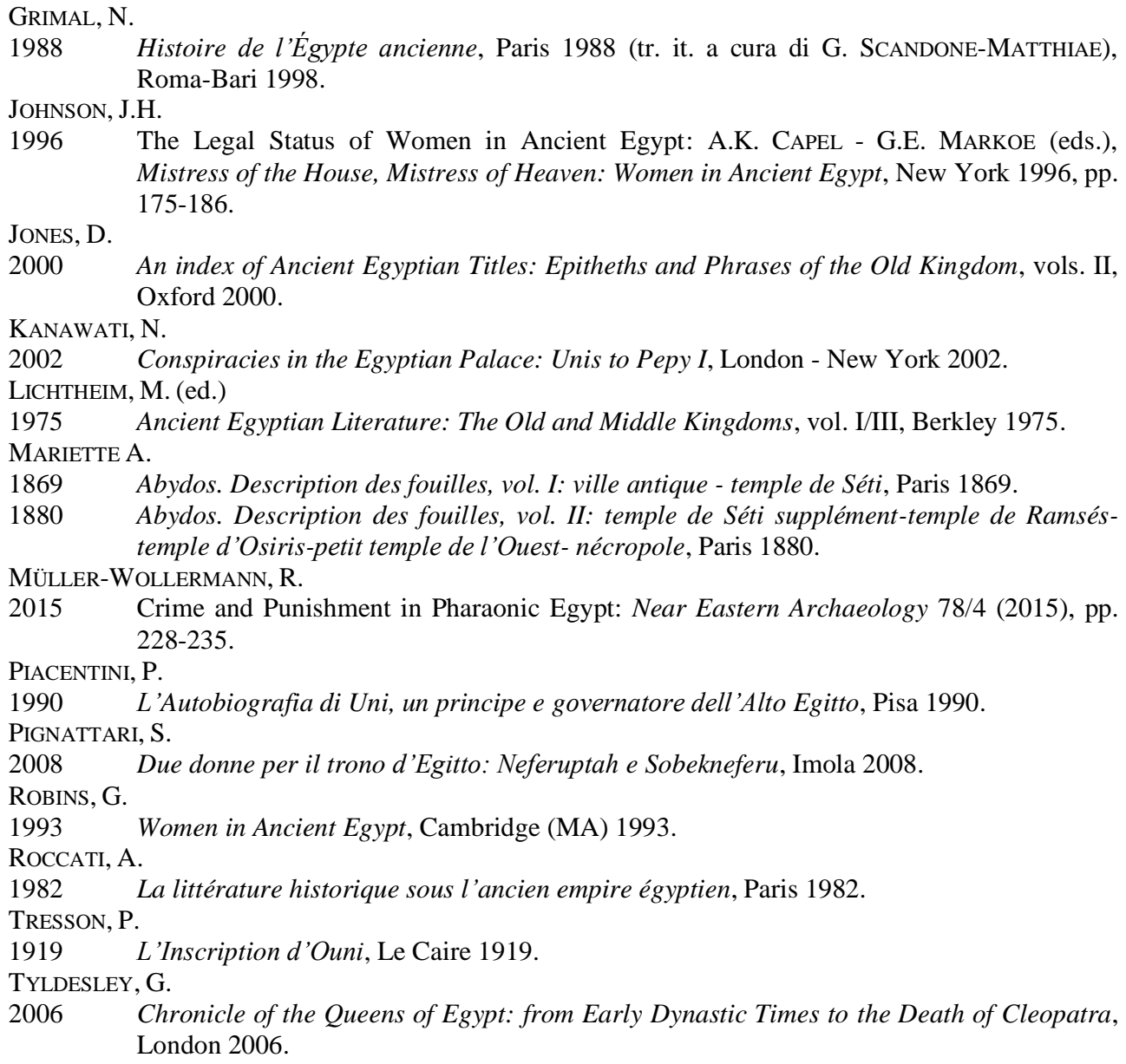




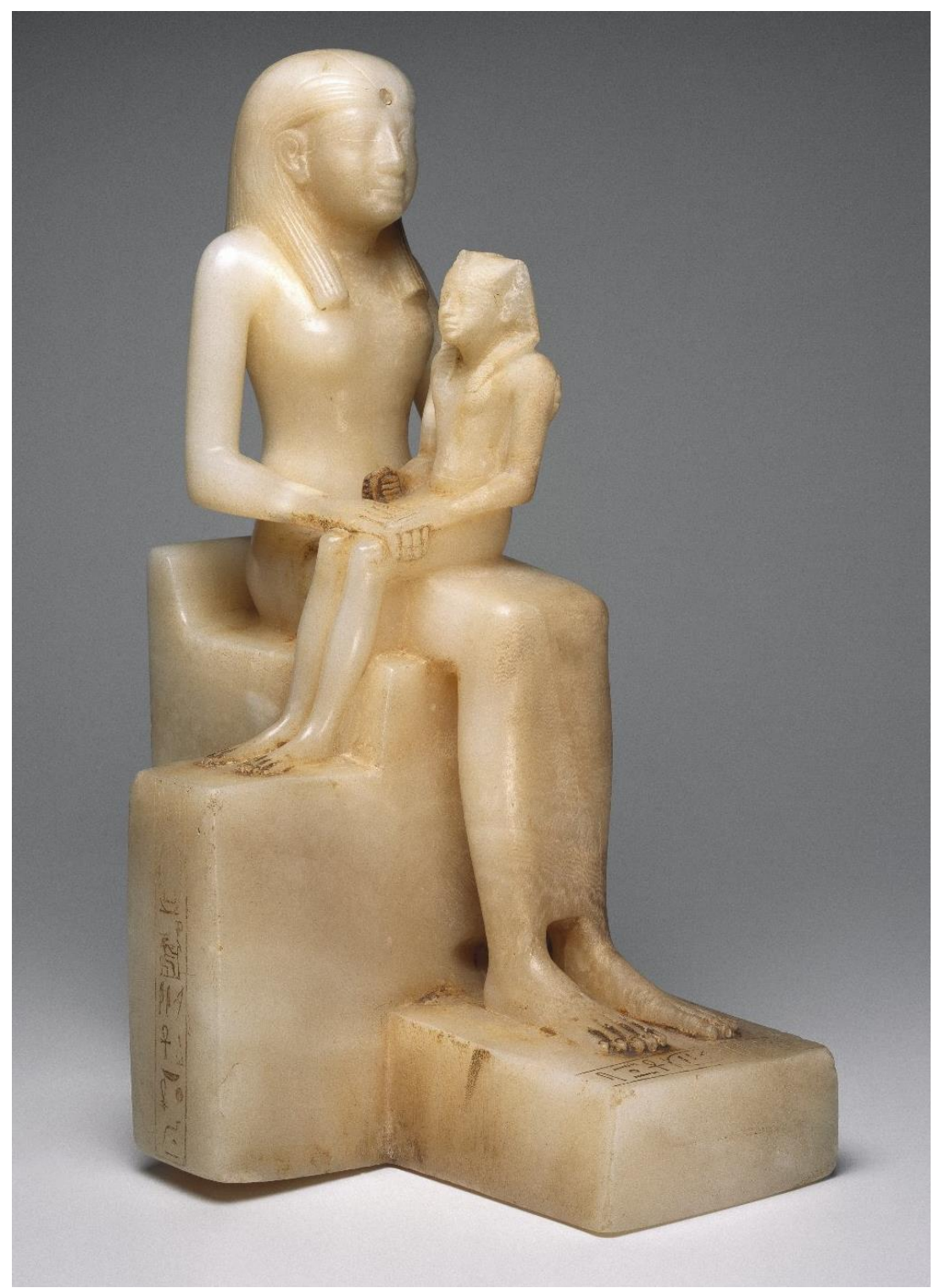

Fig. 1 - Statuetta in alabastro raffigurante la regina Ankhesenpepi II e suo figlio, Pepi II, conservata a New York, Brooklyn Museum (Brooklyn Museum, 39.119_threequarter_right_SL1.jpg; https://www.brooklynmuseum.org/opencollection/objects/3446). 

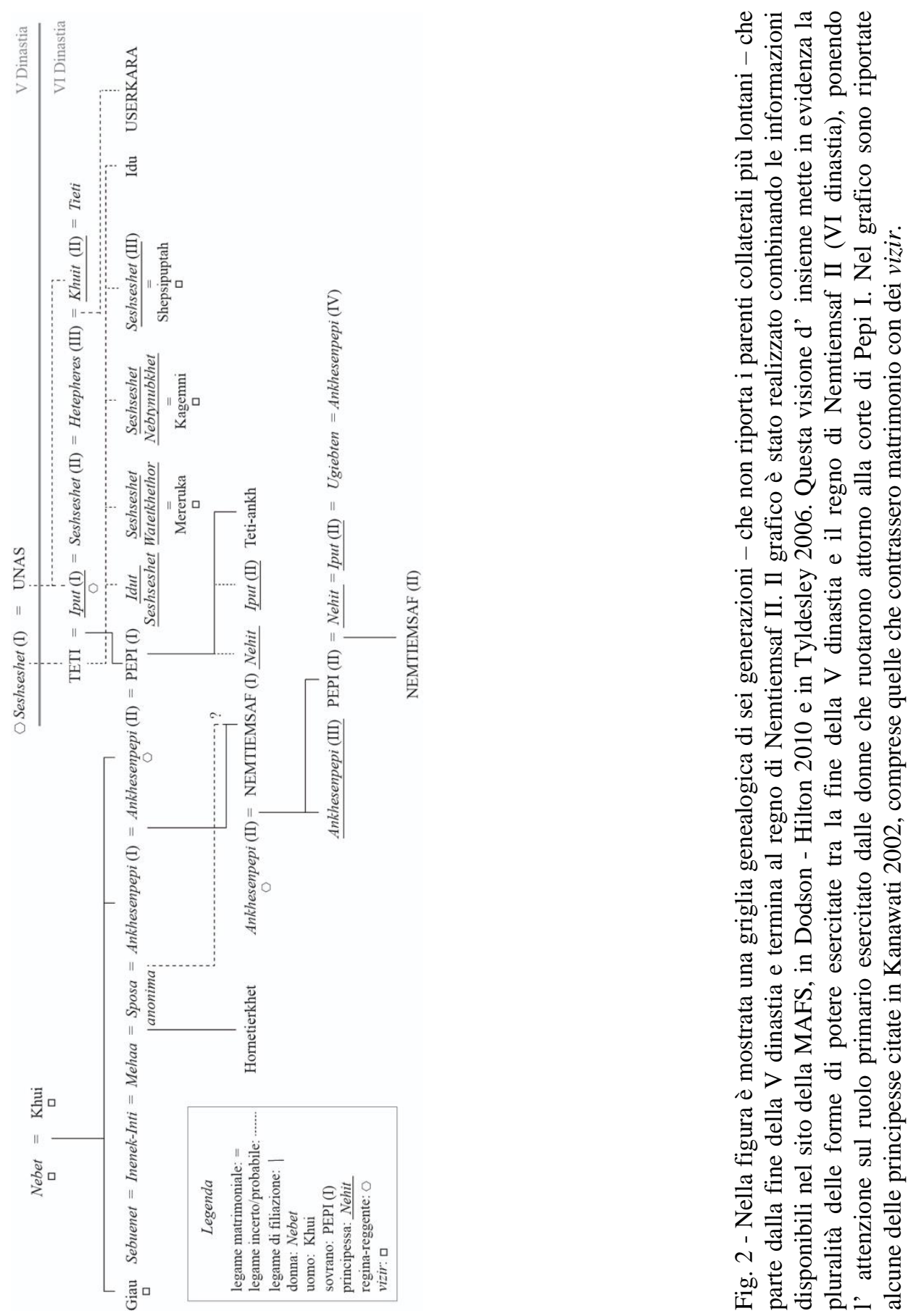